\title{
Study on the tool wear of 3-D elliptical vibration cutting
}

\author{
Jieqiong Lin, Xian Jing, Mingming Lu, Yan Gu, and Jinguo Han \\ Key Laboratory of Micro/Nano and Ultra-precision Manufacturing (Jilin Province), School of Mechatronic \\ Engineering, Changchun University of Technology, Changchun 130012, China \\ Correspondence to: Mingming Lu (lumm@ccut.edu.cn)
}

Received: 26 January 2017 - Revised: 29 May 2017 - Accepted: 16 June 2017 - Published: 11 July 2017

\begin{abstract}
As always, the rapid wear of tools was one of the key factors limiting the precise turning of difficultto-machine materials with diamond tool. 3-D elliptical vibration cutting has inherited many advantages of elliptical vibration cutting, such as the intermittent cutting property and friction reverse property. However, studies on the tool wear of three-dimensional elliptical vibration cutting has not been reported yet. The formation principle of 3-D cutting elliptical trajectory was analysed and a prediction model of tool wear was established in the present work. Besides, a self-developed three-dimensional elliptical vibration device was employed to conduct turning experiment. Compared with the proposed model, the experimental results showed a great agreement with the proposed prediction model. This work may provide a reference for the further optimization of the 3-D elliptical vibration cutting parameters.
\end{abstract}

\section{Introduction}

Since elliptical vibration cutting (EVC) was proposed by Shamoto and Moriwaki (1994), it has been considered as the most potential processing method and has received widespread attention of scholars. A large number of reports have shown that EVC has advantages over many aspects, such as improving the machinability of materials, suppressing the cutting chatter, reducing cutting force, extending tool life and improving the surface uniformity (Jung et al., 2016; Zhou et al., 2016; Zhang et al., 2015, 2016; Li et al., 2015). To date, the studies on EVC are mainly focused on the following aspects: (1) EVC devices, (2) machinability of different materials, (3) EVC cutting force model, (4) tool wear condition.

According to the different driving methods of EVC devices, it can be divided into resonant type and non-resonant type. The resonant type of EVC machining device was developed by E. Shamoto et al. and others (Moriwaki and Shamoto, 1995; Shamoto et al., 2002, 2005; Suzuki, et al., 2003). Driving signals with phase difference were utilized to excite its resonant mode, and an elliptical trajectory could be formed at the tool tip. The machining performance, cutting force model and tool wear resistance of hard and brittle materials were studied in their research. Besides, they pro- posed a 3-D EVC device in 2005. Liu et al. (2004) started to study the resonant type EVC device in 2004. They studied the machinability, ductile-brittle transition model and the effect of tool nose radius on cemented carbide using their proposed device (Ma et al., 2006; Nath et al., 2009a, b). Sathyan et al. studied the resonant EVC device and they also conducted some investigations on chip formation, vibration mark trace and tool wear during the machining process (Ahmed and Sathyan, 2010).

The first non-resonant EVC device was developed by Brehl et al. (1999) Aiming at obtaining the advantage of the developed non-resonant EVC device, they conducted some investigations on the maximum chip thickness, cutting force model, tool wear, and surface quality and so on (Brehl and Dow, 1999). Kim and Loh (2007) studied on non-resonant EVC device since 2007. PCD tool was used to carry out the cutting experiment. A series of structures, such as micro grooves and the pyramid structure, were machined with various elliptical trajectory (Kim and Loh, 2007). In our previous work, an improved memetic algorithm was proposed to achieve nonlinear identification for a 3-D EVC system (Lu et al., 2014).

The analysis of tool wear mechanism for EVC is still limited to 2-D EVC so far. 3-D EVC is developed to satisfy the requirements of cutting surfaces with various shapes and ma- 


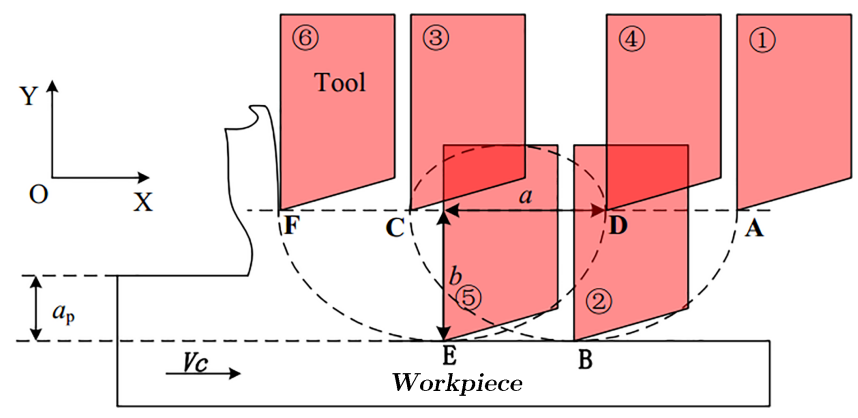

Figure 1. Principle of 2-D EVC.

terials, aiming to achieve the best cutting performances of EVC. The mechanism of 3-D EVC to further improve the tool life has not been reported. In this paper, the reasons for the reduction of tool wear in 3-D EVC are analysed based on the self-developed non-resonant 3-D EVC device. The main structure of this paper is as follows: the principle of 3-D EVC is introduced in Sect.2. Section 3 describes the reduction of tool wear model in 3-D EVC. Section 4 verifies the proposed tool wear model through the experiments.

\section{Principle of 3-D EVC}

3-D EVC is developed from conventional EVC. The remarkable characteristics of EVC are intermittent cutting properties and friction reversal compared with traditional machining. The principle of EVC is shown in Fig. 1, $a$ and $b$ is the semi-major axis and semi-minor axis, respectively. Tool edge is perpendicular to the workpiece. $a_{\mathrm{p}}$ represents the nominal depth of cut, $V_{\mathrm{c}}$ is cutting speed which is generally less than the maximum vibration speed. The tool tip is moving with an ellipse trajectory in XOY plane. As shown in Fig. 1, tool (1) to tool (6) donate to six representative positions of tool tip motion along the elliptical trajectory. Point $\mathrm{A}$ is the starting point of one vibration cycle, point $\mathrm{B}$ is the bottom of the elliptical trajectory, point $C$ is a critical point where tool start to separate from workpiece, and point $\mathrm{D}$ is the starting point of next cycle. The maximum depth of cut and friction reversal occurred at the duration from point $\mathrm{B}$ to point $\mathrm{C}$.

Elliptical vibration cutting has a limitation to turn the complex surfaces. So the 3-D EVC was proposed to address this problem. The principle of 3-D EVC is shown in Fig. 2, ellipse 1 is the projection of tool trajectory on plane YOZ. Take the long axis of ellipse 1 as a rotation axis, the ellipse 2 can be obtained by ellipse 1 rotates a $\beta$ angle. The projection of ellipse 2 on plane XOY and YOZ both are ellipses. And the projection of ellipse 2 on plane $\mathrm{XOZ}$ is a straight line. Take the short axis of ellipse 1 as a rotation axis, the ellipse 3 can be obtained by ellipse 1 rotates a $\alpha$ angle. The projection of ellipse 3 on plane YOZ and XOZ both are ellipses. The elliptical motion trajectory of arbitrary position can be obtained by adjusting the value of angle $\alpha$ and $\beta$. In addition, angle

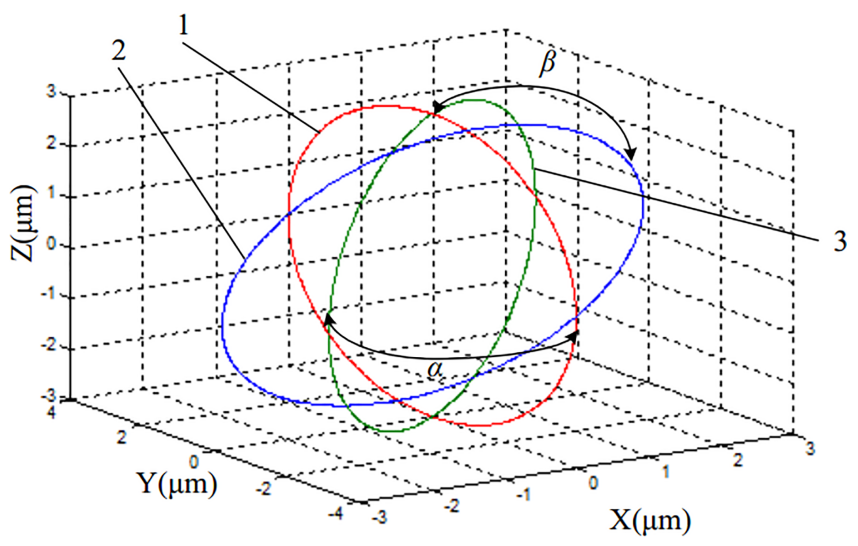

Figure 2. The tool trajectory of 3-D EVC.

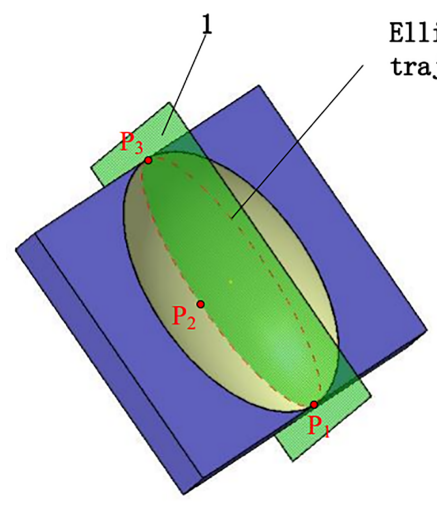

(a) trajectory

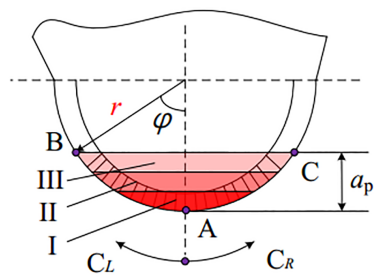

(b)
Elliptical

Figure 3. Wear regions of elliptical vibration cutting.

$\alpha$ and $\beta$ are two important parameters during the modelling process.

\section{Tool wear predictive model of 3-D EVC}

Before the prediction model of tool wear is proposed, it is necessary to introduce the tool wear condition during the elliptical vibration cutting. As shown in Fig. 3a, tool tip pass the point $\mathrm{P} 1, \mathrm{P} 2$ and $\mathrm{P} 3$. At point $\mathrm{P} 2$, tool tip reaches the maximum depth of cut $a_{\mathrm{p}}$. The value of contact area between tool and workpiece is the maximum at this moment. It is assumed that the vibration period of one elliptical vibration cutting is $T$. Then the contact time of tool and workpiece is $t=a_{\mathrm{p}} T /(2 a)$. In this paper, we take it as a severe wear zone when tool tip contact with workpiece at time $[2 t / 3, t]$, and marked it as region I. Similarly, we take it as a moderate wear zone and mild wear zone when tool tip contact with workpiece at time $[t / 3,2 t / 3]$ and time $[0, t / 3]$, and marked it as region II and region III, respectively. We can see from Fig. 3 that tool tip A contact with workpiece at point P1 and P3. The contact marginal point between tool and workpiece turns 

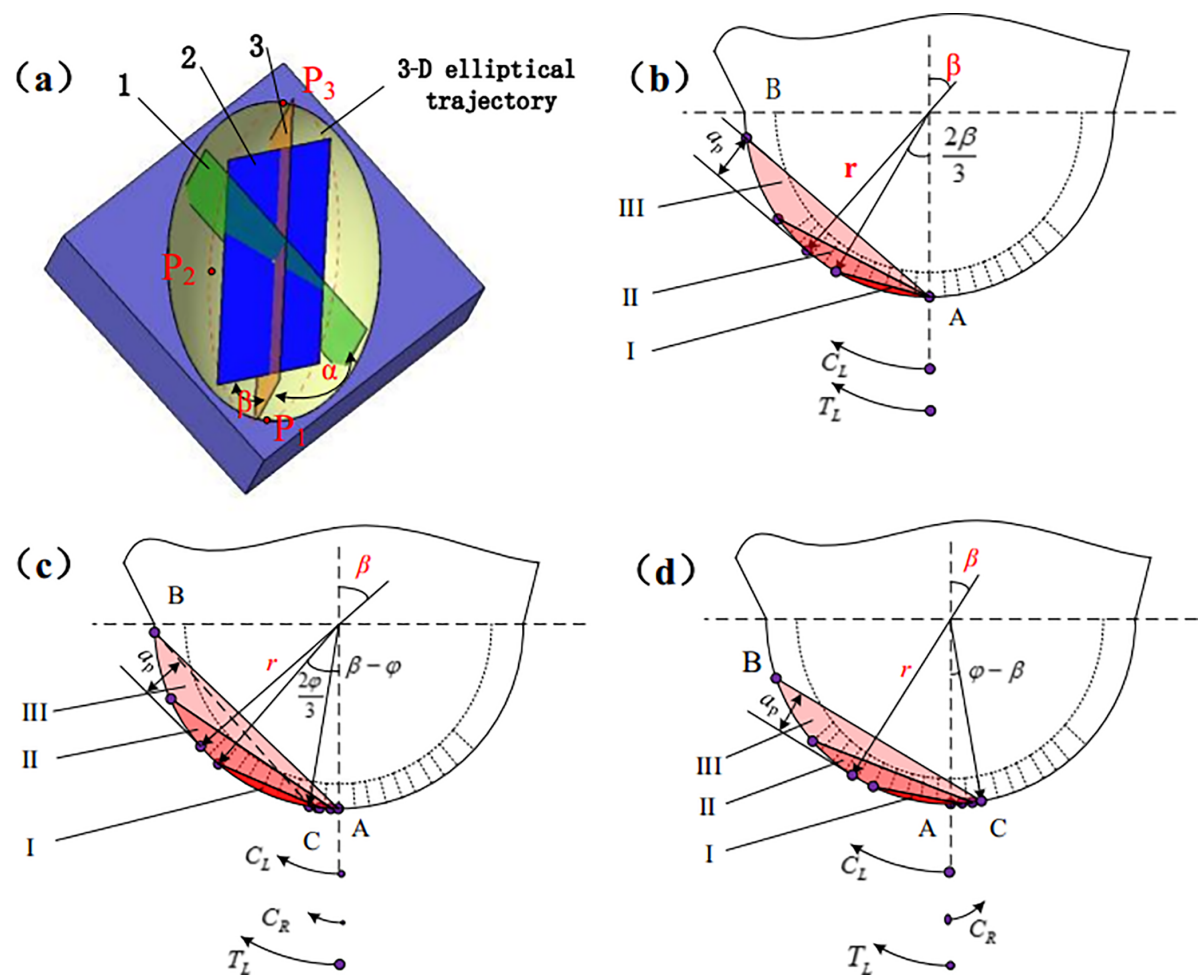

Figure 4. Diagrams of vibration tool trajectory of 3-D EVC and shift of contact marginal points.

Table 1. Machining conditions.

\begin{tabular}{ll}
\hline Parameters & Value \\
\hline Tool material & PCD \\
Tool rake angle $(\mathrm{deg})$ & 0 \\
Tool clearance angle $(\mathrm{deg})$ & 11 \\
Tool nose radius $(\mathrm{mm})$ & 0.2 \\
Workpiece material & Aluminum, brass \\
Feed rate $\left(\mu \mathrm{m} \mathrm{rev}^{-1}\right)$ & 5 \\
Depth of cut $(\mu \mathrm{m})$ & 4 \\
Rotational speed & $15,30,45$ \\
of Spindle $\left(\right.$ rev min $\left.^{-1}\right)$ & \\
Vibration amplitude $(\mu \mathrm{m})$ & $4 \times 4 \times 4(\mathrm{o}-\mathrm{xyz})$, \\
& $4 \times 4(\mathrm{o}-\mathrm{yz})$ \\
\hline
\end{tabular}

to both sides with the increasing of depth of cut. When the depth of cut reaches the maximum value $a_{\mathrm{p}}$, point $\mathrm{B}$ and $\mathrm{C}$ become the contact marginal points. We use $C_{L}$ and $C_{R}$ represent the direction and speed when contact marginal points shift to both sides, respectively.

Tool wear speed reduces to a large extent due to the interrupted cutting characteristic of elliptical vibration cutting. However, tool wear of region I also will affect the cutting accuracy during cutting process. The vibration tool trajectory of 3-D EVC and the shift of contact marginal points are shown in Fig. 4.

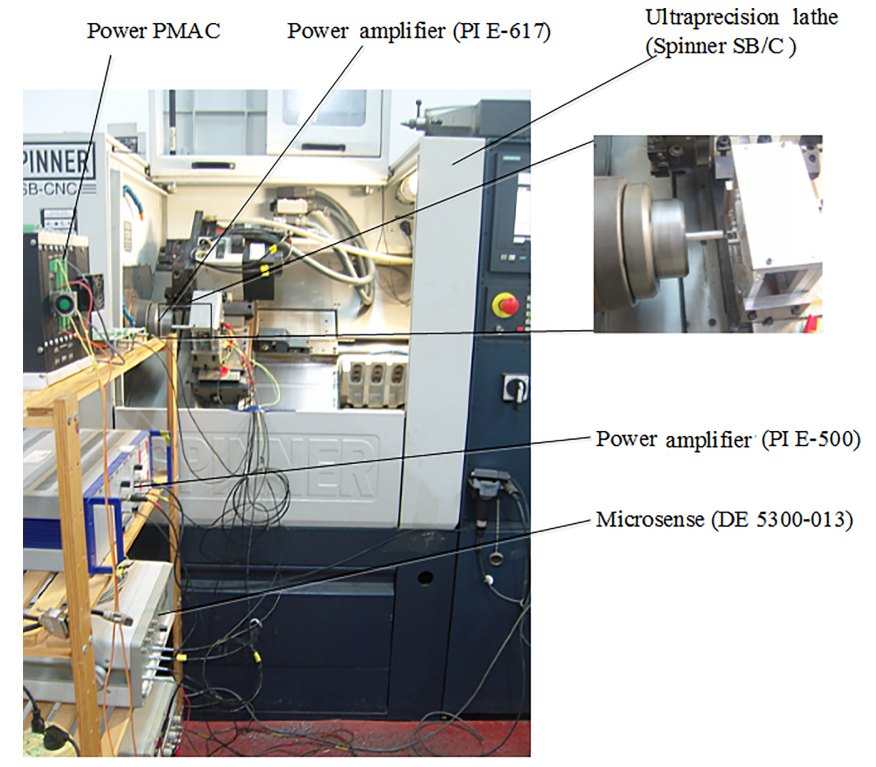

Figure 5. Cutting experiment of 3-D EVC.

As shown in Fig. 4a, the 3-D ellipse trajectory can be obtained by the 2-D ellipse trajectory by a shift of angle $\alpha$ and $\beta$. Point $\mathrm{P}_{1}, \mathrm{P}_{3}$ are donate to the cut in point and cut out point, respectively. Point $\mathrm{P}_{2}$ is the tool tip position relative to workpiece when the depth of cut reaches the maximum value. Angle $\alpha$ mainly affects the position of tool wear. The region 
(a)
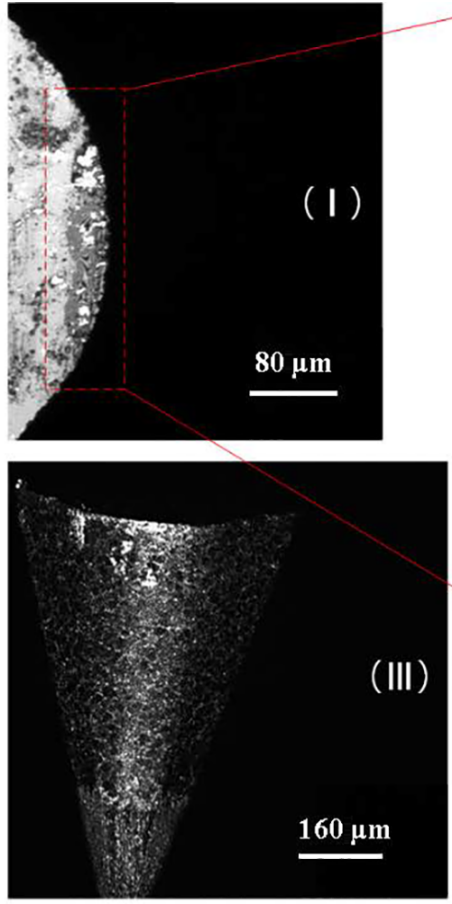

(b)
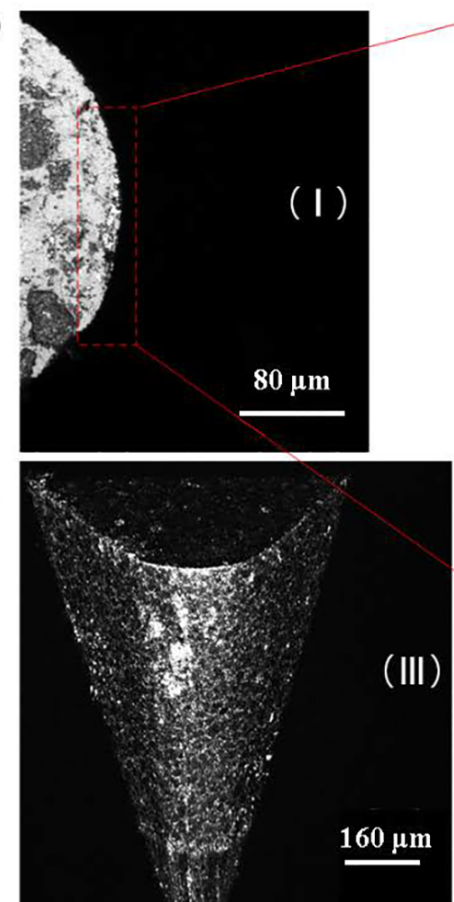

(II)

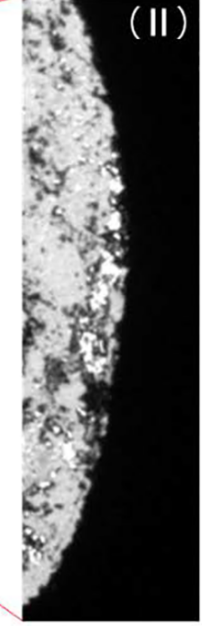

Figure 6. SEM micrographs of tool wear. (a) SEM micrographs of tool using 2-D EVC. (I) Top view of tool tip. (II) Partial enlarged detail of tool tip. (III) Front view of tool. (b) SEM micrographs of tool using 3-D EVC. (I) Top view of tool tip. (II) Partial enlarged detail of tool tip. (III) Front view of tool.

of tool wear locates at the right side of tool nose arc when $\alpha>0$. On the contrary, the region of tool wear located at the left side of tool nose arc when $\alpha<0$. We assume that positive clockwise and negative anti-clockwise. Angle $\beta$ mainly affects the shift speed of contact marginal points between tool and workpiece and the region of tool wear. Thus angle $\alpha$ and $\beta$ have significant influences on tool wear conditions of 3-D EVC.

It is assumed that the tool vibration frequency of 3-D EVC is $f$. Then the tool vibration period is $T=1 / f$. The time to reach the maximum depth of cut is $T a_{\mathrm{p}} /(2 b)$ during cutting. The wrap angle of the maximum tool wear on the one side of tool tip can be obtained as follows:

$\varphi=\arccos \left(\frac{r-a_{\mathrm{p}}}{r}\right)$

On the basis of the arc length formulae $L=\varphi r$, the shift speed of contact marginal points between tool and workpiece can be obtained as follows:

$C_{L}=\frac{2 b L}{T a_{\mathrm{p}}}=\frac{2 b r}{T a_{\mathrm{p}}} \arccos \left(\frac{r-a_{\mathrm{p}}}{r}\right)$

Within the same time $T a_{\mathrm{p}} /(2 b)$, the moving distance of the contact arc central point between tool and workpiece is $L^{\prime}=\beta r$. Then the shift speed of the contact arc central point between tool and workpiece can be expressed as follows,

$T_{L}=\frac{2 b \beta r}{T a_{\mathrm{p}}}$
We analyzed the various tool wear region of 3-D EVC from the following three cases when $\alpha<0$.

When $T_{L}=C_{L}, \beta=\varphi=\arccos \left[\left(r-a_{\mathrm{p}}\right) / r\right]$. The moving speed of the contact arc central point between tool and workpiece is equal to the shift speed of the contact marginal points. That means the contact arc central point $\mathrm{A}$ is coincide with the contact marginal point $\mathrm{C}$ of right side as shown in Fig. 4b. The contact marginal point B of left side is moving at speed of $2 T_{L}$. The tool wear zone was divided into three regions according to the time of tool tip contact with the workpiece. The region $[0,2 \beta / 3]$ of the left side of tool tip is the severe wear region I. The region $[2 \beta / 3,3,4 \beta / 3]$ is the moderate wear region II, and $[4 \beta / 3,2 \beta]$ is the mild wear region III.

When $T_{L}>C_{L}, \beta>\varphi=\arccos \left[\left(r-a_{\mathrm{p}}\right) / r\right]$. The moving speed of the contact arc central point between tool and workpiece is larger than the shift speed of the contact marginal points. That means the contact marginal point $\mathrm{C}$ of right side is shifting to the left slowly from the tool arc central point. The moving speed is $T_{L}-C_{L}$. The moving speed of the contact marginal point $B$ of left side to the left is $T_{L}+C_{L}$. As shown in Fig. 4c, the contact marginal point $\mathrm{C}$ of right side is located at the left of tool tip when tool reaches to the maximum depth of cut $a_{\mathrm{p}}$. The central angle is $\beta-\varphi$ at this moment. The tool wear zone is divided into three regions according to the time of tool tip contact with the workpiece. The region 
$[(2 \beta-2 \varphi) / 3,(3 \beta-\varphi) / 3]$ which is located at the left side of tool tip is region I. Region II located at the left side of tool tip and consists two parts which are $[(\beta-\varphi) / 3,(\beta-2 \varphi) / 3]$ and $[(3 \beta-\varphi) / 3,(3 \beta+2 \varphi) / 3]$. The region $[0, \beta-\varphi / 3]$ and $[(3 \beta+2 \varphi) / 3, \beta+\varphi]$ which are located at the left side of tool tip formed as region III. The contact arc central point A is no longer located at the severe wear region I.

When $T_{L}<C_{L}, \beta<\varphi=\arccos \left[\left(r-a_{\mathrm{p}}\right) / r\right]$. The moving speed of the contact arc central point between tool and workpiece is smaller than the shift speed of the contact marginal points. That means the contact marginal point $\mathrm{C}$ of right side is shifting to the right slowly from the tool arc central point. The moving speed is $C_{L}-T_{L}$. The moving speed of the contact marginal point $\mathrm{B}$ of left side to the left is $T_{L}+C_{L}$. As shown in Fig. $4 \mathrm{c}$, the contact marginal point $\mathrm{C}$ of right side is located at the left of tool tip when tool reaches to the maximum depth of cut $a_{\mathrm{p}}$. The central angle is $\varphi-\beta$ at this moment. The tool wear zone is divided into three regions according to the time of tool tip contact with the workpiece. The region $[0,(\beta+\varphi) / 3]$ on the left side of the tool tip and region $[0,(\varphi-\beta) / 3]$ on the right side of the tool tip formed region I. The region $[(\beta+\varphi) / 3,2 \beta+2 \varphi / 3]$ on the left side of the tool tip and region $[(\varphi-\beta) / 3,(2 \varphi-2 \beta) / 3]$ on the right side of the tool tip formed region II. Similarly, the region $[(2 \beta+2 \varphi) / 3, \beta+\varphi]$ on the left side of tool tip and region $[(2 \varphi-2 \beta) / 3, \varphi-\beta]$ on the right side of tool tip formed region III. The contact arc central point $\mathrm{A}$ is located at severe wear region I.

From the three different tool wear conditions shown in Fig. $4 \mathrm{~b}$ to d, it can be seen that the tool wear can be further reduced by adjusting the cutting parameters during 3-D EVC process compared with the traditional EVC. The verified experiment is shown in Sect. 4.

\section{Results and discussion}

In this paper, a self-developed 3-D EVC device was used to carry out the cutting experiment (Lin et al, 2016). The experiment was carried out on the SPINNER precision lathe. The machining parameters are shown in Table 1 . The machining system is shown in Fig. 5.

The tool vibration frequency used in the experiment is $f=100 \mathrm{~Hz}$. Besides, $\alpha=45^{\circ}, \beta=30^{\circ}$. According to the prediction model of tool wear, we can know that the most serious regions of tool wear for 3-D EVC is located at the left side of tool tip (from front view), which is consistent with the wear condition of Fig. 4d. In this paper, a 2-D EVC experiment was also carried out to offer a comparison. The measurement of tool wear was based on a same cutting distance of workpiece in our experiment. Fifteen workpieces with a diameter of $10 \mathrm{~mm}$ are cut at end faces. The cutting results were compared. As shown in Fig. 6, the regions of tool wear for 2-D EVC are asymmetrically distributed on the both sides of tool tip. The tool wear conditions for 3-D EVC is shown in Fig. $6 b$.

As can be seen from Fig. 6b, it is shown that the tool wear region mainly located at the left side of tool tip (from front view), which is consistent with the condition of Fig. 4d, by adjusting the cutting parameters of 3-D EVC. The wear of tool is smaller than that in 2-D EVC which is verify the correctness of the predictive model. In addition, it also validates that 3-D EVC can reduce the tool wear compared with the 2-D EVC.

\section{Conclusions}

In this paper, a tool wear prediction model was proposed for 3-D EVC. The arc of diamond tool tip was divided into three regions by analysis of the 3-D EVC diamond tool moving mechanism based on the various conditions of the lowest point of tool arc and contact marginal points between tool and workpiece during 3-D EVC cutting. The model was used to predict the tool wear conditions by adjusting the cutting parameters. At last, the tool wear prediction model was verified by experiments. The results indicate that the proposed model is feasible to predict the tool wear of 3-D EVC, which provide theoretical basis for cutting parameters optimization to further extend the tool life.

Data availability. All the data used in this manuscript can be obtained by requesting from the corresponding author.

Competing interests. The authors declare that they have no conflict of interest.

Acknowledgements. This research was financially supported by the Ministry of Science and Technology State Key Support Program (2016YFE0105100), National Natural Science Foundation of China (NSFC) (51375060), Micro-Nano and Ultra-Precision Key Laboratory of Jilin Province (20140622008JC) and Science and Technology Development Projects of Jilin Province (20160520072JH).

Edited by: Bahman Azarhoushang

Reviewed by: two anonymous referees

\section{References}

Ahmed, S. A. and Sathyan, S.: Experimental investigation of transverse vibration-assisted orthogonal cutting of AL-2024, Int. J. Mach. Tool. Manu., 50, 294-302, 2010.

Brehl, D. E., Dow, T. A., Garrard, K., and Sohn, A.: Microstructure fabrication using elliptical vibration-assisted machining (EVAM), ASPE Proceedings, 39, 511-515, 1999. 
Jung, H., Hayasaka, T., and Shamoto, E.: Mechanism and suppression of frictional chatter in high-efficiency elliptical vibration cutting, CIRP Ann.-Manuf. Techn., 65, 369-372, 2016.

Kim, G. D. and Loh, B. G.: Characteristics of chip formation in micro V-grooving using elliptical vibration cutting, J. Micromech. Microeng., 17, 1458-1466, 2007.

Li, W., Lin, F., and Zhang, D.: Study on the improvement of surface quality through ultrasonic elliptical vibration cutting, Advances in Future Manufacturing Engineering: Proceedings of the 2014 International Conference on Future Manufacturing Engineering (ICFME 2014), Hong Kong, 10-11 December 2014, CRC Press, 2, 269-279, 2015.

Lin, J., Lu, M., and Zhou, X.: Development of a Non-Resonant 3D Elliptical Vibration Cutting Apparatus for Diamond Turning, Exp. Techniques, 40, 173-183, 2016.

Liu, K., Li, X. P., Rahman, M., and Liu, X. D.: Study of ductile mode cutting in grooving of tungsten carbide with and without ultrasonic vibration assistance, Int. J. Adv. Manuf. Tech, 24, 389-394, 2004.

Lu, M., Zhou, X., and Lin, J.: Improved Memetic Algorithm for Nonlinear Identification of a 3-D Elliptical Vibration Cutting System, P. I. Mech. Eng. I-J. Sys., 228, 449-460, 2014.

Ma, C. X., Shamoto, E., Xu, L. M., Liu, N., and Moriwaki, T.: UltraPrecision Cutting of Brittle Materials with Ultrasonic Vibrated Diamond Tool[C], Materials Science Forum, Trans Tech Publications, 532, 169-172, 2006.

Moriwaki, T. and Shamoto, E.: Ultrasonic elliptical vibration cutting, CIRP Ann.-Manuf. Techn., 44, 31-34, 1995.

Nath, C., Rahman, M., and Neo, K. S.: Machinability study of tungsten carbide using PCD tools under ultrasonic elliptical vibration cutting, Int. J. Mach. Tool. Manu., 49, 1089-1095, 2009a.
Nath, C., Rahman, M., and Neo, K. S.: A study on the effect of tool nose radius in ultrasonic elliptical vibration cutting of tungsten carbide, J. Mater. Process. Tech., 209, 5830-5836, 2009b.

Shamoto, E. and Moriwaki, T.: Study on Elliptical Vibration Cutting, CIRP Ann.-Manuf. Techn., 43, 38-43, 1994.

Shamoto, E., Suzuki, N., Moriwaki, T., and Naoi, Y.: Development of ultrasonic elliptical vibration controller for elliptical vibration cutting, CIRP Ann.-Manuf. Techn., 51, 327-330, 2002.

Shamoto, E., Suzuki, N., Tsuchiya, E., Hori, Y, Inagaki, H., and Yoshino, K.: Development of 3 DOF ultrasonic vibration tool for elliptical vibration cutting of sculptured surfaces, CIRP Ann.Manuf. Techn., 54, 321-324, 2005.

Suzuki, N., Nakamura, A., Shamoto, E., and Harada, K.: Ultraprecision micromachining of hardened steel by applying ultrasonic elliptical vibration cutting [C], Micromechatronics and Human Science, 2003, MHS 2003, Proceedings of 2003 International Symposium on. IEEE, 221-226, 2003.

Zhang, C., Ehmann, K., and Li, Y.: Analysis of cutting forces in the ultrasonic elliptical vibration-assisted micro-groove turning process, Int. J. Adv. Manuf. Tech., 78, 139-152, 2015.

Zhang, J., Cui, T., Ge, C., Sui, Y., and Yang, H.: Review of micro/nano machining by utilizing elliptical vibration cutting, Int J. Mach. Tool. Manu., 106, 109-126, 2016.

Zhou, X., Zuo, C., Liu, Q., and Lin, J.: Surface generation of freeform surfaces in diamond turning by applying doublefrequency elliptical vibration cutting, Int. J. Mach. Tool. Manu., 104, 45-57, 2016. 